\title{
Measurement and Significance of Three-Dimensional Architecture to the Mechanical Integrity of Trabecular Bone
}

\author{
Steven A. Goldstein, Robert Goulet, Doris McCubbrey \\ Orthopaedic Research Laboratories, Section of Orthopaedic Surgery, University of Michigan, Ann Arbor, Michigan 48109-0486, USA
}

Received: 3 September 1992 / Accepted: 11 February 1993

\begin{abstract}
Summary. The mechanical properties of trabecular bone have been shown to vary significantly with age, anatomic location, and metabolic condition. Efforts towards predicting its behavior have been extensive, and significant relationship between measures of density and mechanical integrity have been reported. Unfortunately, the significant heterogeneity in trabecular bone anisotropy contributes to significant unexplained variance in its strength and modulus when predicted using scalar measures of mass or density. As a result, numerous investigators have attempted to include measures of architecture in an effort to more rigorously investigate potential physiologic optimization strategies, as well as account for the increased fragility associated with advancing age. In our laboratories we have utilized a unique three-dimensional, microcomputed tomography system to measure trabecular plate thickness, trabecular plate separation, trabecular plate number, surface to volume ratio, bone volume fraction, anisotropy, and connectivity in isolated specimens of trabecular bone. The results of these studies demonstrate that in normal bone, more than $80 \%$ of the variance in its mechanical behavior can be explained by measures of density and orientation. The independent measures of connectivity and trabecular plate number were found to be significantly correlated with bone volume fraction, suggesting a potential strategy in the formation of trabecular bone. It might be hypothesized, however, that the relationship between bone volume fraction and connectivity may be substantially altered under conditions associated with aging, fragility, or metabolic bone disease. This hypothesis would be consistent with the histologic evidence of reduced connectivity in osteopenic patients.
\end{abstract}

Key words: Trabecular bone - Density - Mechanical integrity.

For more than a century the observed correspondence between trabecular architecture and whole bone function has been documented as a reflection of a strategy for maintaining mechanical integrity [1-8]. Consequently, compromises in the amount and organization of trabecular bone, as has been associated with aging or metabolic disease, have been implicated as major factors associated with an increased fracture incidence [9-16]. In an effort to quantify the contribution of trabecular bone properties to whole bone integrity, many investigators have performed in vitro biomechanical characterizations of samples of trabecular bone from a variety of

Presented at the NIA Workshop on Aging and Bone Quality, September 3-4, 1992, Bethesda, Maryland

offprint requests to: $\mathrm{S}$. A. Goldstein anatomic locations. The mechanical properties of these specimens (continuum properties, specimens $>5 \mathrm{~mm}$ on any axis) have been shown to vary as much as two orders of magnitude with a dependence on anatomic location, age, and possibly therapeutic agents [9, 17-19]. The distribution of these property variations within the metaphyses correlate well with the specific functional demands placed upon that region. In order to explain these significant variations in trabecular bone mechanical properties, most investigators have made measures of density, mass, or mineral content and correlated these measures to the documented mechanical properties (see $[9,20]$ for review). For in vitro test specimens, both linear and power functions of density have explained nearly $60-70 \%$ of the variance in both modulus and strength $[9,20]$. These results have provided a rational basis for the use of noninvasive techniques for estimating trabecular bone properties. As illustrated in Figure 1, measures of apparent bone density, for $8 \mathrm{~mm}$ cubic specimens of trabecular bone, correlate well with measured modulus values. However, a significant proportion of the variance of the data is not explained by a measure of density alone. As has been noted on numerous occasions in the literature, this finding is not surprising as it attempts to use a scalar quantity to explain anisotropic or directionally dependent mechanical properties $[4,9,16,19,21-23]$. Clearly, the architecture or organization of the trabecular tissue within the specimens must contribute to its inherent mechanical properties. Similarly, it would be expected that alterations in the architecture and morphology of trabecular bone, such as the loss in connectivity and number of trabeculae associated with osteopenia, would significantly contribute to an increased fracture risk in anatomic regions composed primarily of trabecular bone and subjected to high loads.

The three-dimensional architecture of trabecular bone has been described as being composed of a series of interconnected plates or rods with a gross porosity greater than $30 \%[24,25]$. Numerous methods have been described for characterizing its two- and three-dimensional architectural features utilizing stereologic-algorithms or direct threedimensional digitization $[4,7,16,21,22,25-28]$. These techniques have enabled investigators to begin establishing relationship between the patterns of trabecular architecture and physiologic function or disease state. The incentive for many investigators has been to either determine which features of trabecular morphology are most critical to providing mechanical integrity or to test hypotheses regarding possible optimization strategies that have evolved. At the University of Michigan we have been fortunate to have access to a unique microcomputed tomography scanning system for the evaluation of trabecular bone. In general, the microcomputed tomography system provides a three-dimensional digitization of small specimens at resolutions that depend on 


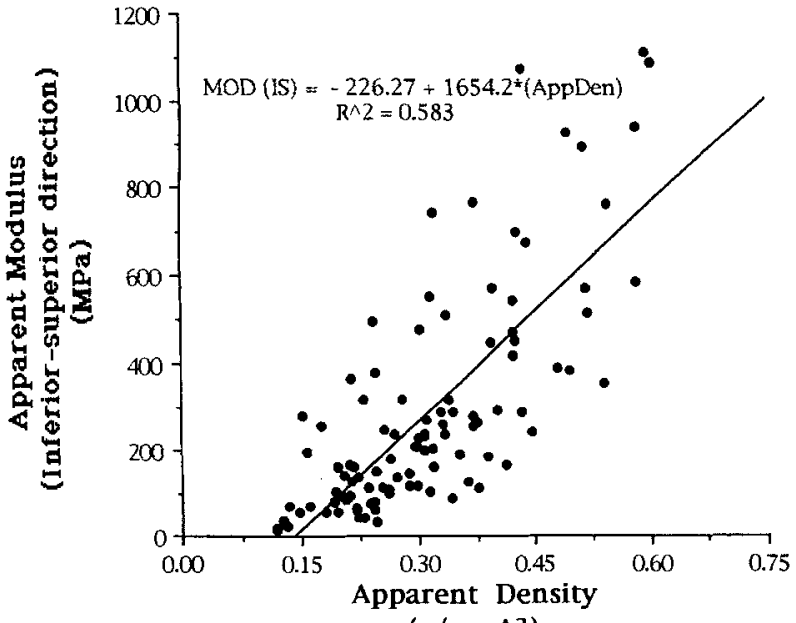

$\left(\mathrm{g} / \mathrm{mm}^{\wedge} \mathrm{3}\right)$

Fig. 1. The relationship between apparent density and modulus for $8 \mathrm{~mm}$ cubic specimens of trabecular bone extracted from several human metaphyseal regions is illustrated. Note that while a significant relationship was found, a large amount of variance in the data remains unexplained. This graph was generated using data taken from the work of Goulet et al. [22, 30].

specimen size, but typically range between 10 and $70 \mu \mathrm{m}$. Typical reconstructed digitizations of several trabecular bone cube specimens are illustrated in Figure 2. The details of the instrumentation, theory, and algorithms associated with this system and comparisons to alternative techniques have been published elsewhere [21, 23, 29, 30].

It is the purpose of this paper to present a review of data collected in our laboratories which might provide insight concerning the contribution of specific trabecular bone architectural features to its mechanical behavior. Two specific research programs are reviewed. The first set of studies were designed to determine the statistical relationships between trabecular structure and mechanical integrity in human metaphyseal bone, and the second research program was designed to investigate the consequences of estrogen deficiency on the structural and mechanical properties of trabecular bone.

\section{The Relationship Between Structure and Mechanical Integrity in Human Metaphyseal Bone}

\section{Continuum Trabecular Properties}

One hundred and four $8 \mathrm{~mm}$ cubic specimens of trabecular bone were scanned on the microcomputed tomography system. The specimens were obtained from four fresh frozen cadavers aged 31-69 years (2 females 55 and 61 years, 2 males 31 and 69 years), from seven metaphyseal regions, with a range in bone volume fraction from 6 to $36 \%$.

For each scanned specimen a series of morphologic and architectural measures were calculated [21, 23, 30]. After appropriate thresholding utilizing a locally adaptive image processing algorithm to produce a binary image of bone and non-bone voxels, measures of bone volume fraction, trabecular plate number, trabecular plate thickness, trabecular plate separation, and bone surface to bone volume ratio were calculated. In addition, the anisotropy of the specimens was determined utilizing a three-dimensional representation of the method of secants to produce measures of orientation

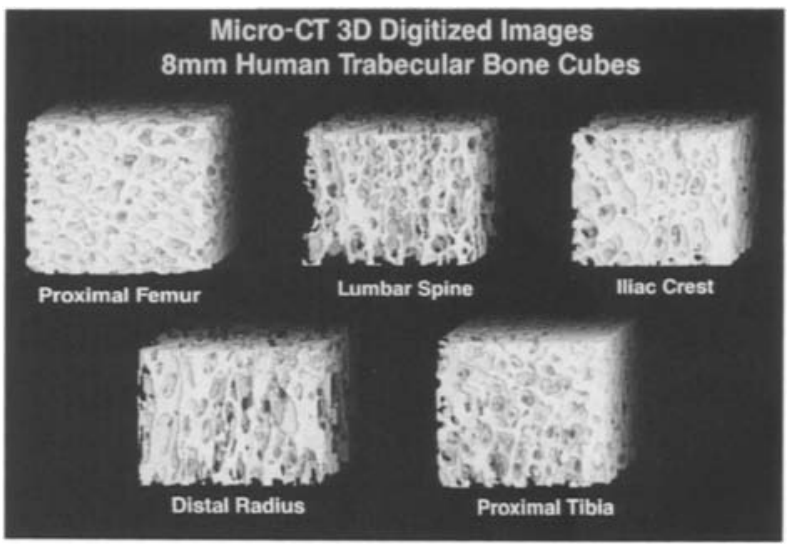

Fig. 2. A typical reconstructed image of trabecular bone samples using the microcomputed tomography system is illustrated. The unique system provides a complete three-dimensional digitization with the resolution dependent on magnification. The images shown represent digitizations with $50 \times 50 \times 50 \mu \mathrm{m}$ voxels.

and degree of orientation. Finally, a measure of connectivity was made by calculating the Euler-Poncare number for the three-dimensional images, based on an expansion of the methodology described by Serra [31]. A complete description of the calculations for the connectivity measure can be found in Feldkamp et al. [21]. It is important to note that the measures of degree of anisotropy and connectivity contain no information regarding the dimensions of the connected or oriented trabecular struts, or the strength of the material comprising the connections. In addition, there is no scale imposed on the measures of connectivity or degree of anisotropy.

After scanning, each specimen was then subjected to orthogonal mechanical testing in a Instron materials testing system. Preyield tests were conducted in the three orthogonal directions followed by a test to failure in one randomly chosen direction. Measures of tangent elastic modulus and ultimate strength were made for each specimen. Finally, the bone samples were evaluated for apparent and ash weight density. The details of these procedures can be found elsewhere $[19,30]$.

The results of these studies demonstrated several important statistically based structure function relationships. A significant relationship among apparent density, ash density, and bone volume fraction was found with $90 \%$ of the variance explained. This suggests that the measure of bone volume fraction is representative of measures of mineral content for these isolated specimens. More importantly, a number of interesting correlations were found among the morphologic variables. Three of the morphologic variables are independent measures with no mathematical interrelationships: trabecular plate number, bone volume fraction, and connectivity. Results of the multiple regression analyses demonstrated a highly significant relationship for trabecular plate number and connectivity with bone volume fraction (Figs. 3-4). As demonstrated, these results suggest that for a given amount of trabecular bone, a predetermined number of interconnections and number of trabecular struts are formed. These results would suggest that the evolution and development of trabecular bone follows and that measures of bone volume fraction would be sufficient to also estimate the number of trabeculae and their connectivity. It would also be expected, therefore, that statistically based formulas relating mechanical properties to morphologic features would only 


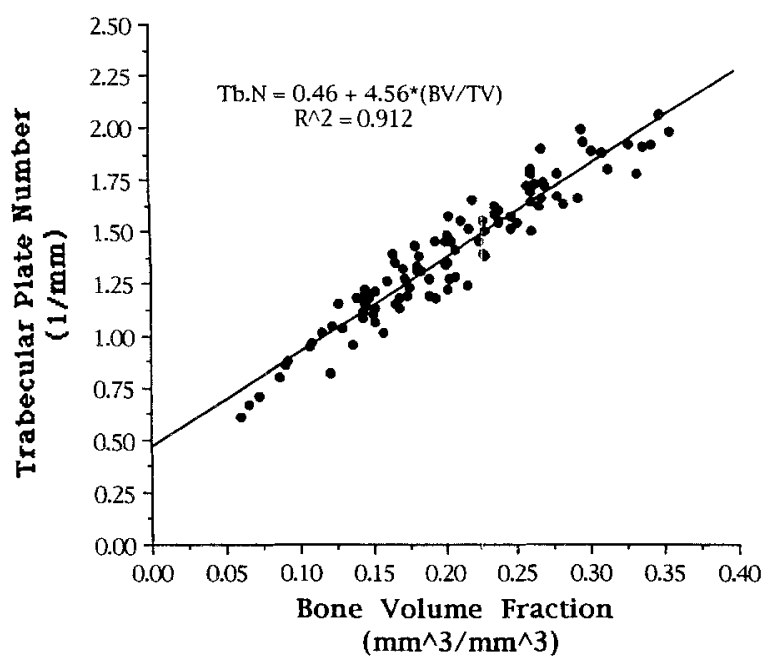

Fig. 3. A statistically significant relationship between bone volume fraction and trabecular plate number was found for the human metaphyseal specimens evaluated. (Data taken from studies of Goulet et al. $[22,30]$.)

need to include a measure of bone volume fraction in a normal population. In an effort to explain the mechanical behavior of trabecular bone, multiple regression models were evaluated, and it was found that the directional elastic moduli of a trabecular bone specimen could be predicted by measures of bone volume fraction and anisotropy. This empirical relationship was able to explain approximately $90 \%$ of the variance in the mechanical data for modulus and strength, and as expected, was not really improved by the addition of measures of connectivity or trabecular plate number. It is therefore reasonable to conclude that the mechanical behavior of trabecular bone can be almost entirely explained by accounting for how much bone is present and its orientation. This dependence on organization can be demonstrated by the example illustrated in Figure 5. Two specimens of nearly identical bone volume fraction are illustrated along with maps representing an assessment of their orientation. The top specimen is very anisotropic, as illustrated by its elliptical mean intercept length profile, whereas the bottom specimen is reasonably isotropic, as illustrated by its spherical mean intercept length profile. Despite having the same bone volume fractions (or amount of bone mass), the directional moduli varied by an order of magnitude.

Though the data presented would seem to suggest that much of the behavior of trabecular bone can be explained by measures of anisotropy and bone volume fraction, two critical issues need to be addressed. First, though the selected specimens for evaluation represented a wide variation in bone volume fraction, metaphyseal location and to a lesser degree, age, the study population did not include what could be represented as histomorphometrically confirmed osteoporotic bone. It is reasonable to hypothesize that significant metabolic diseases as well as specific treatment modalities might alter the apparent morphologic strategy determined from these specimens. In other words, the relationship between connectivity and bone volume fraction may be altered by disease or therapeutic agents. In this case, the relationship between connectivity and volume fraction might be diagnostic of a bone disorder and would suggest that measures other than anisotropy and volume fraction might need to be considered to explain mechanical behavior or predict risk of fracture.

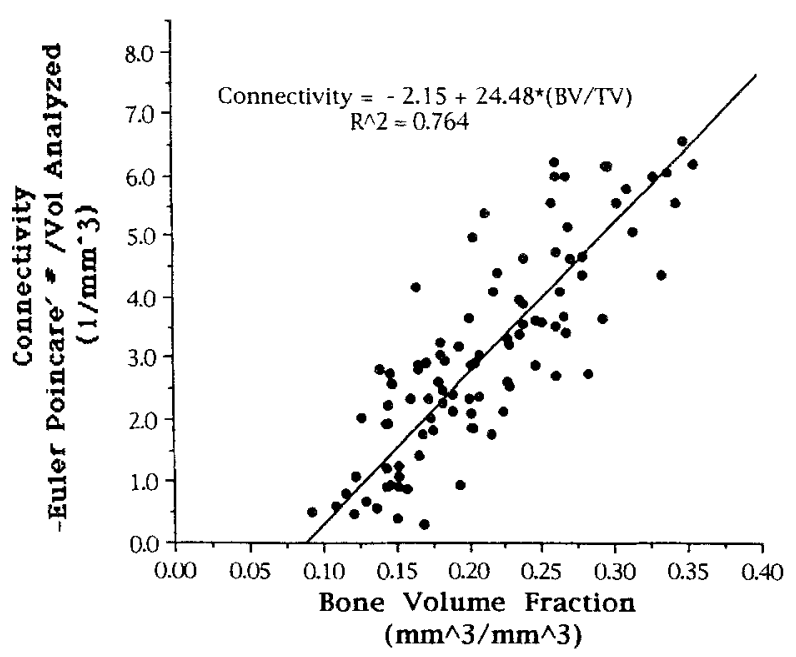

Fig. 4. A significant relationship was also found between the independent measures of bone volume fraction and connectivity (-EulerPoincare number). This finding suggests that in normal metaphyseal trabecular bone, a specific organizational strategy has evolved (Data redrawn from Goulet et al. $[22,30]$.)

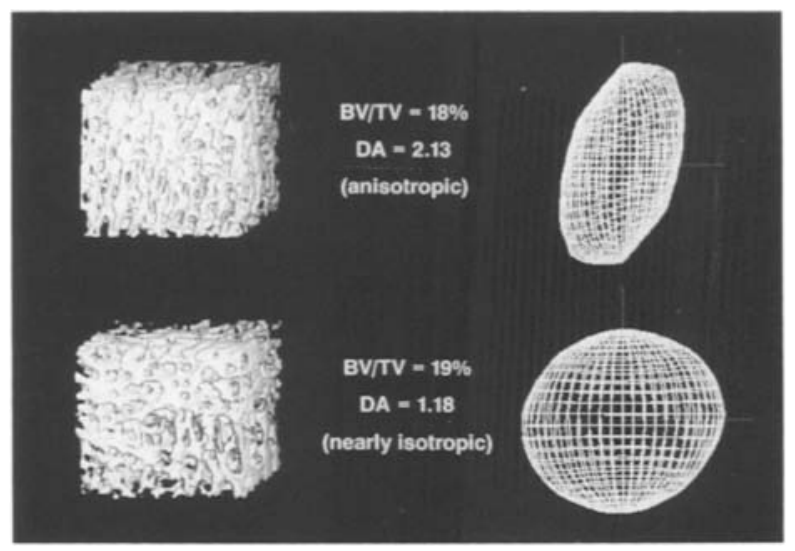

Fig. 5. The effect of trabecular orientation on mechanical properties is illustrated in this figure. The two bone specimens have nearly identical bone volume faction, but significantly different orientation of trabeculae. The orthogonal mechanical properties of these two specimens varied from $30 \%$ in the inferior/superior direction to more than an order of magnitude in the anterior/posterior direction.

\section{Evaluation of Trabecular Tissue Properties}

Inherent in the relationships between the architecture and mass of the trabecular bone and its mechanical behavior is the assumption that the tissue that forms the trabeculae has similar material properties across regions and individuals. Clearly, it may be possible that the effects of aging, metabolic disease, or its treatment may alter the constituent properties of the extracellular matrix of bone or its organization. These alterations may be detectable as changes in the mechanical integrity of individual trabeculae.

In a series of published studies, we have presented unique techniques for testing standardized specimens of bone machined from individual trabeculae. Utilizing a specially designed micromilling machine we were able to create parallelopiped beams of trabecular tissue of typical dimensions $(100 \times 100 \times 1500 \mu \mathrm{m})$. These beams were then tested 
Table 1. Morphologic and mechanical properties of vertebral trabecular bone in control ( $\mathrm{SH})$, ovariectomized (OV), and ovariectomized with calcitonin (CA) treatment in beagles, 12 months postsurgery

\begin{tabular}{lllllllll}
\hline Group & $\begin{array}{l}\text { BV/TV } \\
\left(\mathrm{mm}^{3} / \mathrm{mm}^{3}\right)\end{array}$ & $\begin{array}{l}\text { BS/TV } \\
\left(\mathrm{mm}^{2} / \mathrm{mm}^{3}\right)\end{array}$ & $\begin{array}{l}\text { Tb.Th } \\
(\mathrm{mm})\end{array}$ & $\begin{array}{l}\text { Tb.S } \\
(\mathrm{mm})\end{array}$ & $\begin{array}{l}\text { Tb.N } \\
(1 / \mathrm{mm})\end{array}$ & $\begin{array}{l}\text { Connectivity } \\
\left(1 / \mathrm{mm}^{3}\right)\end{array}$ & $\begin{array}{l}\text { Ultimate } \\
\text { load } \\
(\mathrm{N})\end{array}$ & $\begin{array}{l}\text { Number cycles } \\
\text { to failure }\end{array}$ \\
\hline SH & $0.425 \pm 0.019$ & $15.947 \pm 1.364$ & $0.127 \pm 0.010$ & $0.173 \pm 0.018$ & $3.370 \pm 0.313$ & $26.735 \pm 6.265$ & $929.6 \pm 246.9$ & $48.4 \pm 24.9$ \\
OV & $0.430 \pm 0.029$ & $15.451 \pm 0.470$ & $0.130 \pm 0.004$ & $0.174 \pm 0.017$ & $3.304 \pm 0.145$ & $25.034 \pm 3.661$ & $564.9 \pm 159.3$ & $25.6 \pm 16.5$ \\
CA & $0.471 \pm 0.046$ & $12.452 \pm 1.014$ & $0.176 \pm 0.027$ & $0.196 \pm 0.032$ & $2.857 \pm 0.183$ & $18.438 \pm 2.246$ & $351.4 \pm 53.1$ & $17.4 \pm 12.4$ \\
\hline
\end{tabular}

$\mathrm{SH} \longrightarrow$ sham, OV—ovariectomy, CA—ovariectomy and calcitonin $(8 \mu \mathrm{g} / \mathrm{kg} / \mathrm{day})$

The morphology data in this table was acquired from analysis of a standardized region of vertebral (L2) trabecular bone extracted from the right superior quadrant (approximate volume, $6 \mathrm{~mm}^{3}$ ). The mechanical properties were determined from monotonic and fatigue tests of whole vertebra (L3 and L4)

in 3 or 4 pt. bending, and by including precise dimensional measures and assuming isotropic properties we were able to calculate an apparent moduli for each specimen. We have conducted both monotonic and fatigue tests. The details of these procedures can be found in the papers by [32-34]. Perhaps one of the most interesting findings from these studies was the significant variability in tissue modulus. In an effort to explain the variation in these properties, we calculated the relative density of the specimens and found that this measure of mineral content could only explain approximately $40 \%$ of the variance in the modulus. These results suggest that properties at this hierarchy of trabecular tissue are dependent on architecture, mineral profile, and tissue constituency. It should be noted that at this magnification, the architectural features would include such measures as number of osteocyte lacunae, orientation, and width of lamellae, number of trabecular packets, packet volume, and perhaps, collagen matrix orientation. It seems plausible, however, that significant alterations might occur within this hierarchy, influencing the properties of the trabecular tissue. Some of these alterations have been demonstrated by other investigators [10] demonstrating an age-related increase in lamellar thickness in females, and age-related decrease in packet size in males and females. Further evidence is provided by a pilot study conducted in our laboratory. Single trabecular microbeams were machined from lumbar vertebrae of a 30- and 36-year-old cadaver as well as an 80- and 87-year-old cadaver and tested in micro four point bending for modulus determination. As demonstrated in Figure 7, we found a statistically significant reduction in tissue modulus from bones from the older cadavers. Though this study is extremely preliminary in nature, it further supports the plausibility that significant alterations may be occurring at the tissue or extracellular matrix level as a function of age, metabolic disease, and potentially therapeutic intervention.

\section{Effect of Estrogen Deficiency on Trabecular Bone Architecture and Mechanical Behavior}

In a study designed to evaluate the effects of estrogen deficiency on trabecular bone in a canine model, we have had the opportunity to further evaluate the relationship between architecture and mechanical properties [35]. In retired breeder beagles (age 3-9, mean age 6.5 years) one group of animals were ovariohysterectomized (OV) and then euthanized 12 months later in order to characterize the effects of estrogen deficiency on trabecular bone properties $(n=5)$. A sham $(\mathrm{SH})$ control group followed the same protocol $(\mathrm{n}=5)$. The effects of treatment with calcitonin (CA) $(8 \mu \mathrm{g} / \mathrm{kg} / \mathrm{day})$ in a third group of $\mathrm{OV}$ animals were also evaluated $(\mathrm{n}=5)$. Lumbar vertebrae (L2) from the animals were dissected free and scanned on the microcomputed tomography system in an effort to quantify trabecular bone morphology and architecture. After scanning, whole vertebrae (L3-L4) were tested to failure in an MTS servohydraulic testing system in order to measure whole bone properties. The results after 12 months suggested that no statistically significant alterations occurred in bone morphology or architectural variables demonstrated trends supported by other investigators [36-38], it is possible that the sample sizes were too small for these to be significant (Table 1). However, despite the small sample size, there was a statistically significant decrease in the mechanical properties (load to failure and number of cycles to failure) for the whole vertebrae when comparing the $\mathrm{OV}$ to the SH control animals and a further decrease in the CA animals (Table 1). In addition, the relationship between connectivity and bone volume fraction was found to be significantly decreased for the CA group when compared with the $\mathrm{SH}$ and OV groups. As seen in Figure 6, this relationship was unchanged in the face of the ovariectomy alone. These surprising findings suggest that the significant reductions in mechanical properties due to ovariectomy in the canine model is not dependent on alterations in trabecular bone morphology or orientation. Although it might be argued that a small loss in connectivity may cause a devastating decrease in mechanical integrity, the data to date do not support this hypothesis. As noted earlier, it was hypothesized that trabecular bone structure function relationships might be affected by therapeutic agents. Clearly, this hypothesis is supported by a variation in the correlation between volume fraction and connectivity (Fig. 6). In addition, although the vertebral cortical properties were not directly quantified, evaluation of geometric and cortical material properties from the humerus, tibia, and femur showed no significant alterations; this suggests that changes in mechanical properties were likely not due to alterations in cortical bone in these animals.

The results of this study would suggest that because no significant alteration in architecture or morphology was found due to estrogen deficiency alone, that factors other than bone volume and architecture contributed to the reduction in whole bone mechanical behavior. One possible explanation for these findings would be that the properties of the trabecular tissue comprising the individual struts was altered in the face of estrogen deficiency. At this hierarchy, this might be referred to as the quality of the trabecular tissue. However, as these animals were only followed for 12 


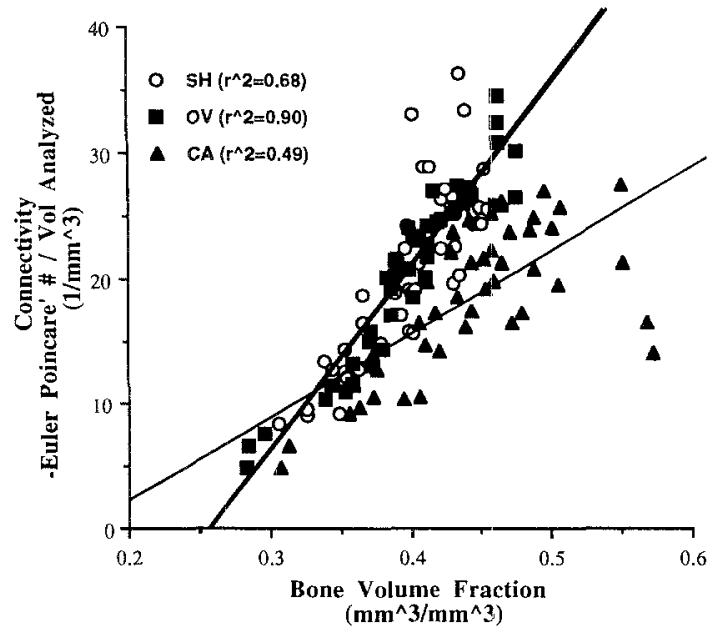

Fig. 6. The relationship between bone volume fraction and connectivity is demonstrated for vertebral (L2) trabeculae bone in canines that were sham operated (SH), after ovariectomy (OV), and with ovariectomy and calcitonin (CA) treatment $(8 \mu \mathrm{g} / \mathrm{kg} /$ day). Though the relationship between the morphologic measures was unaffected after ovariectomy, treatment with calcitonin significantly altered this fundamental morphologic relationship. (Data taken from McCubbrey et al. [35].)

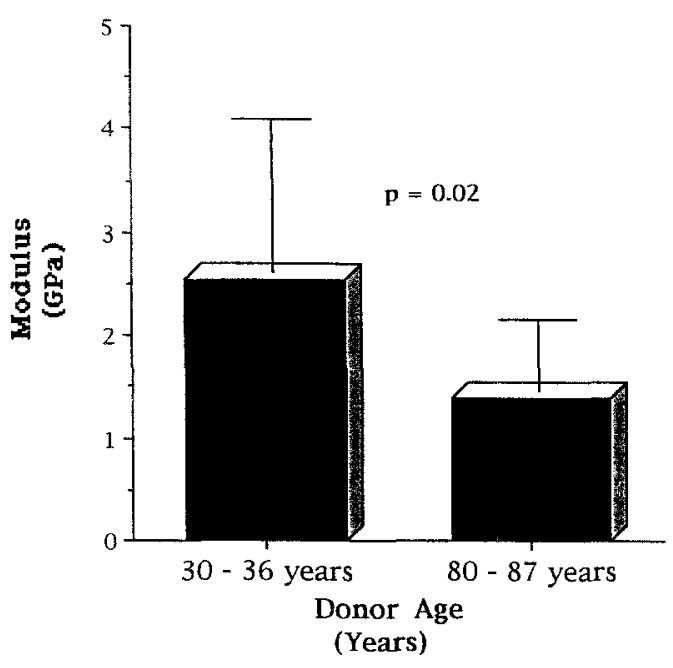

Fig. 7. The results from a preliminary study investigating the properties of trabecular tissue from elderly (ages 80 and 87 ) and relatively young (ages 30 and 36 ) cadavers is demonstrated. These preliminary data suggest that significant alterations might occur during the process of aging, resulting in reduced material property for the tissue that constitutes trabecular bone.

months, it may be unlikely that a major portion of the trabecular tissue was remodeled. Perhaps the most plausible explanation may relate to potential effects on the surface of the trabeculae. From a material science point of view, any defects or imperfections in the surface of individual trabeculae (remodeling site), may significantly reduce their resistance to crack initiation and propagation.

\section{Summary and Future Directions}

Though we have gained some insight into the potential con- tribution of architecture and/or connectivity of trabecular bone to its mechanical properties, there are many issues that remain to be investigated. It may be reasonable to generalize that the mechanical integrity of trabecular bone is dependent on four factors: bone volume fraction or density, orientation, connectivity, and tissue properties (properties of the extracellular matrix). It is also reasonable to assume that one or more of these factors might be significantly effected by aging, metabolic disease, or therapeutic intervention. Perhaps what is clear are specific questions which must be addressed if we are to advance our understanding of factors that might contribute to trabecular properties or the generalized fragility of bone.

First, we need to determine the effects of age on the relationships between measures of mass and organization. For example, are the mechanisms associated with trabecular structural optimization age-dependent?

Second, it is critical to determine the influence of experimental loading conditions on the predictions of continuum properties based on morphologic and architectural measures. In other words, due to the complex anisotropic, inhomogeneous behavior of trabecular bone, it is likely that the bone has been optimized for response to a specific set of loading characteristics. What is the response of trabecular bone to cyclic or fatigue loads? Perhaps it may be more important to more carefully evaluate the post-yield characteristics of trabecular bone, which may be instructive in determining its mechanisms of failure.

Finally, we need to determine the contribution of "tissue properties" to the integrity of trabecular bone and the effects of aging on these properties. It will be critical to determine whether age, disease, or therapeutic intervention alter the mechanisms associated with the microarchitectural construction of the extracellular matrix or whether these conditions affect the individual constituent properties of the matrix components. Once this pathway is determined it may be more feasible to target the cellular mechanisms responsible or affected.

Acknowledgments. This research was supported by NIH Grants AR34399, AR41349, AG07542. We would also like to thank Mike Ciarelli, Kuiwon Choi, and Anita Grierson for their assistance and Drs. Janet Kuhn, Jeff Bonadio, Lee Feldkamp, and Mike Parfitt for their advice and collaboration.

\section{References}

1. Brand R, Claes L (1989) Review: the Law of bone remodeling by Julius Wolff. J Biomech 22(2):185-187

2. Carter DR, Orr TE, Fyhrie DP (1989) Relationships between loading history and femoral cancellous bone architecture. J Biomech 22(3):231-244

3. Cowin SC (1986) Wolff's law of trabecular architecture at remodeling equilibrium. J Biomed Eng 108:83-88

4. Hayes WC, Snyder B (1981) Toward a quantitative formulation of Wolff's law in trabecular bone. In: Cowin SC (eds) Mechanical properties of bone. ASME, pp 43-68

5. Koch JC (1917) The laws of bone architecture. Am J Anat 21: $177-298$

6. Lanyon, LE (1974) Experimental support for the trajectorial theory of bone structure. J Bone Jt Surg 56B(1):160-166

7. Raux P, Townsend PR, Miegel R, Rose RM, Radin EL (1975) Trabecular architecture of the human patella. J Biomech $8: 1-7$ 
8. Roux W (1895) Gesameltz Abhandlungen uber der Entwicklungsmechanik der Organisman. Leipsiz, W. Engelmann

9. Goldstein SA (1987) The mechanical properties of trabecular bone: dependence on anatomical location and function. J Biomech 20:1055-1061

10. Kragstrup J, Melsen F, Mosekilde L (1983) Thickness of bone formed at remodelling sites in normal human iliac trabecular bone: variations with age and sex. Metab Bone Dis Rel Res 4:291-295

11. Lips P, Courpron P, Meunier PJ (1978) Mean wall thickness of trabecular bone packets in human iliac crest: changes with age. Calcif Tissue Res 26:13-17

12. McCubbrey DA, Goldstein SA, Cody DD, Goulet RW, Kuhn JL (1991) The regional density, architectural, and tissue properties of vertebral trabecular bone and their relation to whole bone failure properties, Adv Bioeng 20:575

13. Mosekilde L (1989) Sex differences in age-related loss of vertebral trabecular bone mass and structure: biomechanical consequences. Bone 10:425-432

14. Parfitt AM, Mathews CHE, Villaneuva AR, Kleerekoper M, Frame B, Rao DS (1983) Relationships between surface, volume, and thickness of iliac trabecular bone in aging and in osteoporosis. J Clin Invest 72:1396-1409

15. Parfitt AM (1984) Age-related structural changes in trabecular and cortical bone: cellular mechanisms and biomechanical consequences. Calcif Tissue Int 365:123-128

16. Snyder BD (1991) Anisotropic structure-property relations for trabecular bone. Ph.D. Thesis, University of Pennsylvania, Philadelphia, PA, 1991

17. Brown TD, Ferguson AB (1980) Mechanical property distributions in the cancellous bone of the human proximal femur. Acta Orthop Scand 51:429-437

18. Carter DR, Hayes WC (1977) The compressive behavior of bone as a two-phase porous structure. J Bone Joint Surg 59m A(7):954 962

19. Ciarelli MJ, Goldstein SA, Kuhn JL, Cody DD, Brown M (1991) The orthogonal mechanical properties and density of human trabecular bone from the major metaphyseal regions utilizing materials testing and computed tomography. J Orthop Res 9: 674-682

20. Rice JC, Cowin S, Bowman JA (1988) On the dependence of elasticity and strength of cancellous bone on apparent density. $J$ Biomech 21:155-168

21. Feldkamp LA, Goldstein SA, Parfitt AM, Jesion G, Kleerekoper M (1989) The direct examination of three-dimensional bone architecture in vitro by computed tomography. $\mathbf{J}$ Bone Miner Res 4:3-11

22. Goulet RW, Ciarelli MJ, Goldstein SA, Kuhn JL, Feldkamp LA, Kruger D, Viviano D, Champlain F, Matthews LS (1988) The effects of architecture and morphology on the mechanical properties of trabecular bone. Trans 34th Orthop Res Soc 13:73

23. Kuhn JL, Goldstein SA, Feldkamp LA, Goulet RW, Jesion G (1990) Evaluation of a microcomputed tomography system to study trabecular bone structure. J Orthop Res 8:833-842

24. Singh I (1978) The architecture of cancellous bone. J Anat 127: 305-310

25. Whitehouse WJ (1974) The quantitative morphology of anisotropic trabecular bone. J Microsc 101(2): 153-168

26. Merz WA, Schenk RK (1970) Quantitative structural analysis of human cancellous bone. Acta Anat 75:54-66

27. Odgaard A, Jensen EB, Gundersen HJG (1990) Estimation of structural anisotropy based on volume orientation. A new concept. J Microscopy 157:149-162

28. Pugh JW, Radin EL, Rose RM (1974) Quantitative studies of human subchondral cancellous bone. J Bone Joint Surg [Am] 56-A(2):313-321

29. Kuhn JL, Goulet RW, Goldstein SA, Feldkamp LA (1988) A study of variation of trabecular architectures in small volumes of bone using a microcomputed tomography system. Proc 12th American Soc of Biomechanics Meeting, Urbana-Champaign, Illinois, pp 16-17

30. Goulet RW, Goldstein SA, Ciarelli MJ, Kuhn JL, Brown MD, Feldkamp LA (in press) Relationship between the structural and orthogonal mechanical properties of trabecular bone. J Biomech
31. Serra J (1982) Image analysis and mathematical morphology. Academic Press, London

32. Kuhn JL, Goldstein SA, Choi K, London M, Feldkamp LA, Matthews LS (1989) A comparison of the trabecular and cortical tissue moduli from human iliac crests. J Orthop Res 7:95-107

33. Choi K, Khun JL, Ciarelli MJ, Goldstein SA (1990) The elastic moduli of human subchondral, trabecular, and cortical bone tissue and the size-dependency of cortical bone modulus. J Biomech 23:1103-1113

34. Choi K, Goldstein SA (1992) The fatigue properties of human trabecular bone tissue. J Biomech 25(12):1371-1381

35. McCubbrey DA, Yian EH, Goulet RW, Shih MS, Parfitt AM, Goldstein SA (1993) The effects of calcitonin on trabecular bone properties in the ovariectomized beagle. Transactions of the 1993 Orthopaedic Research Society Meeting, February 1993

36. Faugere MC, Friedler RM, Fanti P, Malluche HH (1990) Bone changes occurring early after cessation of ovarian in beagle dogs: a histomorphometric study employing sequential biopsies. J Bone Miner Res 5:263-272

37. Martin RB, Butcher RL, Sherwood LL, Buckendahl P, Boyd RD, Farris D, Sharkey N, Dannicci G (1987) Effects of ovariectomy in beagle dogs. Bone 8:23-31

38. Malluche HH, Faugere MC, Rush M, Friedler R (1986) Osteoblastic insufficiency is responsible for maintenance of osteopenia after loss of ovarian function in experimental beagle dogs. Endocrinology 119:2649-2654

39. Vesterby A, Gundersen HJG, Melsen F (1989) Star volume of marrow space and trabeculae of the first lumbar vertebra: sampling efficiency and biological variation. Bone 10:7-13

\section{DISCUSSION}

DR. GRYNPAS: When you talk about the tissue level property, would it make a difference if you had a single trabecula with a lot of variation in mineralization, i.e., microheterogeneity? Would that make it weaker or stronger?

DR. GOLDSTEIN: I can't tell you what is the most effective characteristic of the mineral that would make it better from a mechanical integrity point of view. But without question the profile of the mineral plays an important role. The features I think important are the profile of the mineralization, the specific architecture of the packets, the properties of the individual lamellae, and the connections between them (whether there are loose collagen or cement lines between the packets, et cetera). All play a role, but we have no idea how any of them contribute to mechanical properties at this point.

DR. MONIER-FAUGERE: It is very interesting that you found a decrease in the compressive strength in the vertebrae of the oophorectomized dog. I recently found that there was a decrease in bone volume, a decrease in the number of trabecular and a decrease in trabecular thickness in the vertebrae of the beagle dog, 18 months after oophorectomy. Perhaps you didn't wait long enough.

DR. GOLDSTEIN: At 12 months there really should be a robustness of the experimental problem. You may have to look at a lot of dogs.

We observed barely a trend for trabecular plate thickness and bone volume fraction to decrease. But although these were borderline significant, the mechanics were highly sig- 
nificant. That is what gives me confidence that there are other factors.

DR. MONIER-FAUGERE: How many dogs did you use per group?

DR. GOLDSTEIN: There were five per group.

DR. MONIER-FAUGERE: That is definitely not enough.

DR. BIEWENER: I was surprised by the extremely low density values you have on the individual mechanical test trabeculae. They ranged from 0.2 to 0.4 percent, which is considerably below cortical bone. Could you explain?

DR. GOLDSTEIN: Those were not true density values. Those are a dimensionless, relative measure of mineral den- sity, dependent on the attenuation of the high resolution $\mathrm{X}$-ray source.

DR. BIEWENER: The stiffness of those trabeculae were also considerably lower than what you might expect.

DR. GOLDSTEIN: That is an important point. The reason the stiffness is lower is because the physiologic or the anatomic/histologic features now become significant stress risers. That is reality. When you work down to that level, a lacunae can occupy about 30 microns. That is a big hole, a big stress riser compared to a specimen that is only 100 microns or so in size. That may be what we are dealing with, and why those properties are low. When we did a size evaluation in cortical bone, going from specimens that were 100 microns all the way up to a millimeter in size, we saw a very significant relationship with the surface-to-volume ratio. So there is no question there is a size effect. 